\title{
Relación de la Transmitancia Atmosférica con la Heliofanía y la Diferencia de Temperaturas Extremas Diarias en la Zona Ecuatorial Andina
}

\author{
Celso Recalde ${ }^{(1)}$, César Cisneros ${ }^{(2)}$, Diego Vaca ${ }^{(3)}$ y Cristina Ramos $^{(3)}$ \\ (1) Escuela Superior Politécnica de Chimborazo, Facultad de Ciencias, Panamericana Sur Km 1 1/2, \\ Riobamba-Ecuador (e-mail: crecalde672000@yahoo.com) \\ (2) Instituto Superior Politécnico José Antonio Echeverría, Centro de Estudios de Tecnologías Energéticas \\ Renovables Habana-Cuba (e-mail:, cesar@ceter.cujae.edu.cu) \\ (3) Instituto Nacional de Eficiencia Energética y Energías Renovables, Av. 6 de Diciembre N33-32 e Ignacio \\ Bossano, Quito-Ecuador (e-mail: diego.vaca@iner.gob.ec, cristina.ramos@iner.gob.ec)
}

Recibido Jun. 3, 2014; Aceptado Jul. 22, 2014; Versión final recibida Sep. 13, 2014

\begin{abstract}
Resumen
En este trabajo se proponen valores de transmitancia atmosférica, utilizando la heliofanía (horas de sol) y la diferencia de temperaturas extremas diarias de una localidad de la zona ecuatorial alto andina. Para tal efecto, se calculó la radiación total extraterrestre estudiándose la confiabilidad de los modelos de energía solar total incidente. Luego se realizó un tratamiento estadístico de los valores atípicos a las variables de radiación total incidente, heliofanía y temperaturas máximas y mínimas diarias en la ciudad de Riobamba, las mismas que fueron medidas por casi cinco años desde junio del 2007. Con estos datos, se calcularon los coeficientes de correlación lineal entre las variables utilizando tres modelos: uno que usa la heliofanía y dos que utilizan las diferencias de temperaturas, observándose que existe una relación más consistente entre la radiación y la heliofanía. Además se estableció que no es adecuado generalizar los valores obtenidos para otras regiones, aunque tengan condiciones atmosféricas similares.
\end{abstract}

\section{Relationship between Atmospheric Transmissivity with Daily Heliophany and Extreme Temperature Difference in the Andean Equatorial Zone}

\begin{abstract}
In this work, values of atmospheric transmittance coefficients using heliophany (sunshine hours) and the difference between maximum and minimum temperatures are proposed for a city located in the Andean Ecuadorian zone. First, the extraterrestrial radiation was calculated to study the reliability of the models. Later, statistical techniques were applied to meteorological data measured since June 2007 for a period of almost five years. These data were used to calculate the empirical lineal coefficients between different variables, using three models: one using heliophany and two using the difference between temperatures. The results showed that there is a more consistence relation between solar radiation and sunshine hours. In addition, it was established that it is not adequate to generalize the results to other locations even if they have similar weather characteristics.
\end{abstract}

Keywords: atmospheric transmittance, solar radiation, extraterrestrial solar radiation, Andean Ecuador 


\section{INTRODUCCIÓN}

Desde épocas ancestrales en la zona andina se desarrollaron conocimientos sobre el comportamiento de los cuerpos celestes, siendo el Sol (Inti), la estrella más cercana a la que orientaron mayor esfuerzo (Recalde et al., 2010). La radiación solar incidente en la Tierra es la principal fuente de energía renovable puesto que da inicio a la cadena energética global y es la variable más importante en meteorología. Como fuente de energía de la mayoría de los procesos en nuestro planeta, es esencial en los procesos físicos, químicos y biológicos como la fotosíntesis que influye en la evapotranspiración, crecimiento de los cultivos, entre otros (Sayago et al., 2011); también es un parámetro primordial para la construcción de modelos y simulaciones en diversos campos de las ciencias, ingenierías, e incluso en las nuevas corrientes como biofísica y biotecnología (Recalde et al., 2013) con aplicaciones en salud, biodiversidad (Magnani et al., 2009), dimensionamiento de sistemas energéticos, (Monne et al., 2011; Lema et al., 2005) estimaciones meteorológicas, efectos globales, entre otros (Lara et al., 2013; Machado et al., 2010). Desafortunadamente, existe poca información disponible a nivel local y global debido a que se requieren grandes inversiones para el equipamiento, medición, tratamiento y registro de la radiación solar, aproximadamente sólo en una de cada 500 estaciones meteorológicas se realizan observaciones de radiación solar incidente (Raichijk et al., 2005). Para suplir esta falta de información, se han desarrollado numerosos intentos por estimarla en una amplia gama de escalas espaciales y temporales (Sayago et al., 2011).

En el caso particular del Ecuador existe un manifiesto interés en las aplicaciones energéticas del sol. Por este motivo es importante al menos estimar la radiación solar incidente puesto que la misma define los diseños e instalaciones de los dispositivos fotovoltaicos y termosolares. Para aprovechar la energía solar fotovoltaica se han definido dos grandes direcciones: sistemas conectados a la red y sistemas autónomos para su uso en regiones alejadas de las redes de distribución de energía eléctrica (Rodríguez y Sarmiento, 2010). La energía termosolar por otro lado, desarrolla sus aplicaciones energéticas a baja, media y alta temperatura. La calidad de radiación solar incidente en un dispositivo de aprovechamiento solar depende entre otros aspectos: de la difusión de la radiación solar en la atmosfera, de la reflectancia del suelo y de la fracción visible del cielo en el punto de interés. Los sistemas de energía solar son más eficientes en condiciones despejadas que bajo condiciones nubladas; incluso, la mayoría de los dispositivos solares operan correctamente solo bajo condiciones de cielo despejado, de aquí la importancia de evaluar el recurso solar considerando las condiciones de cada sector, siendo la generación y gestión energética un reto, en particular en la zona rural, más aun en la región andina por su irregular topografía (Peixóto y Oort, 1984).

La duración y la cantidad de radiación solar dependen de la combinación de efectos astronómicos y meteorológicos. El potencial energético solar tiene una fuerte dependencia de parámetros como la altura, el ángulo de inclinación, la orientación del dispositivo de captación y principalmente las características atmosféricas y climatológicas reinantes. En este trabajo se analiza el caso de la zona ecuatorial alto andina que presenta zonas geográficas con irregularidad topográfica, a gran altura hasta los $6200 \mathrm{msnm}$. Muchos trabajos han tratado de determinar la disponibilidad del recurso solar hallando las variaciones que experimenta la radiación solar incidente cuando aumenta la altura (Salazar et al., 2007). En Ecuador, por su posición geográfica, el recurso solar es importante pero no existen bases de datos históricas de radiación, lo que dificulta su óptimo aprovechamiento particularmente en sitios de altura. Cualquier correlación o modelo para sitios de altura que se desee determinar debe contemplar los efectos de la nubosidad, la polución y otros factores que afecten a la radiación solar incidente (Igbal, 1983). De la física atmosférica se sabe que a medida que aumenta la altura, la atmósfera se hace más tenue, por lo que el valor de la radiación debe ser mayor que en sitios menos altos (Igbal, 1983). Al considerar cielo claro, de igual manera la fracción difusa debe disminuir precisamente al bajar la densidad atmosférica. Sin embargo, no es práctico basar las predicciones sobre cálculos de la radiación solar solo sobre la atenuación de la radiación extraterrestre por la atmosfera. Para la predicción del comportamiento de los procesos solares, se usan mediciones históricas de radiación solar en la localidad y/o de localidades cercanas similares (Gray et al., 2010; Vernich y Zuanni, 1996). Por estas razones se analizaron los parámetros meteorológicos que se monitorean con más frecuencia en la red de estaciones de la zona alto andina ecuatorial, se buscaron los modelos que mejor se adapten a esta información y que se hayan aplicado en regiones que por sus características puedan ser comparados con los resultados del presente trabajo, los coeficientes de los modelos de transmitancia atmosférica mostraron buena consistencia en la validación.

\section{METODOLOGÍA}

En el año 2007 se instaló una estación meteorológica automática en la zona ecuatorial, a una altura de 2840 m.s.n.m., hemisferio sur, zona 17 9816967,05N 758398,29E, en el campus politécnico de la ciudad de Riobamba, espacio regular, libre de obstrucciones externas y accesible para el monitoreo y mantenimiento. Las mediciones de radiación solar global incidente se tomaron con un piranómetro Li-Co \#LI-200SA, con 
certificado de calibración y error típico del $5 \%$ los datos se recolectaron cada diez minutos entre Junio del 2007 hasta abril del 2012 y se almacenaron en un data logger NRG Symphonie. Por otro lado, a unos 950 metros está la estación meteorológica convencional del Instituto Nacional de Meteorología e Hidrología del Ecuador denominada "Riobamba Politécnica" No 14 Código MA10, de donde se obtienen entre otros datos: duración del brillo solar o heliofanía, la presión atmosférica, temperatura, humedad relativa, punto de rocío, nubosidad, precipitación, velocidad y dirección de viento que son archivadas manualmente según las horas sinópticas 07h00, 10h00, 13h00, $16 \mathrm{~h} 00$ y 19h00. Se trabajaron las mediciones de temperatura máxima, temperatura mínima y heliofanía.

Considerando que las corrientes marinas bosquejan un ciclo climático de aproximadamente cuatro años y sus efectos son más significativos que los de un ciclo solar, se dividió los datos en dos grupos, uno entre los años 2009, 2010 y 2011 para obtener los coeficientes de la regresión lineal de los modelos, información que representan aproximadamente el $73 \%$ del total y un segundo grupo de datos que corresponde a los años 2007, 2008 y 2012 que se empleó para la validación. Se realizaron análisis de consistencia detectando valores atípicos con las pruebas de datos anómalos univariantes, bivariantes y multivariantes por métodos gráficos y de máxima y mínima kurtosis, con los datos tomados de las dos estaciones meteorológicas, identificando errores de los procesos de validación basados en horas de brillo solar y diferencias diarias de temperaturas extremas, se obtuvieron los coeficientes de regresión para la ciudad de Riobamba (Peña, 2002) y se les comparó con los propuestos por otros autores.

En este documento se trabajó con tres modelos empíricos propuestos a partir de la definición de transmitancia atmosférica $\mathrm{T}$, que es una medida de la atenuación de la radiación solar extraterrestre, se puede calcular por la ecn. (1), donde $\mathrm{H}$ representa los valores de la radiación solar incidente en la superficie terrestre medida por un piranómetro y $\mathrm{H}_{\mathrm{o}}$ la radiación solar extraterrestre calculada.

$\tau=100 \frac{\mathrm{H}}{\mathrm{H}_{\mathrm{o}}}$

En Ecuador la atenuación de la radiación solar por la atmosfera es influenciada, tanto en espacio como en tiempo por las corrientes marinas, la fría de Humboldt, la cálida del Niño, los grandes anticiclones semi permanentes en el Atlántico y en el Pacifico, el cambio de posición a lo largo del año de las áreas de alta presión, las alturas de Bolivia, la zona ecuatorial y la cordillera de los Andes (Baigorria et al., 2004). Otro factor que podría cambiar la transmitancia atmosférica son los fenómenos naturales imprevistos. En el caso de la zona andina del Ecuador la ceniza volcánica presente en la atmósfera producto de las erupciones principalmente del volcán Tungurahua, ubicado aproximadamente a $35 \mathrm{~km}$ en línea recta de las estaciones meteorológicas, sin embargo, se considera despreciable por ser una actividad esporádica y principalmente debido a que el movimiento de la ceniza fluctúa con la velocidad y dirección del viento. En este estudio se consideró que su incidencia no afecta de manera significativa y permanente a la transmitancia atmosférica; en cuanto a las características ambientales de la localidad, la ciudad de Riobamba tiene como principal actividad económica el comercio con pocas pequeñas industrias, con un bajo efecto del polvo y ceniza por lo que se supone una atmósfera relativamente limpia.

Se requiere ajustar la base de datos meteorológicos a la hora solar y de aplicar la definición de transmitancia atmosférica descrita por la ecn (1), para esto a la hora local se adiciona dos correcciones la primera que consiste en cuatro minutos por cada grado de diferencia entre el meridiano estándar que afecta al lugar analizado $-78,7^{\circ}$ y el meridiano de la zona horaria local $-75^{\circ}$ y la segunda, correspondiente al aporte de la ecuación del tiempo mostrada en la ecn. (2), que considera las perturbaciones producidas por la rotación terrestre (Igbal, 1983; Villeda et al., 2011).

$E=229,2(0,000075+0,001868 \cos B-0,032077 \sin B-0,014615 \cos 2 B-0,04089 \sin 2 B)$

El día del año se determina con la variable B desde la ecn. (3), donde $n$ representa los 365 días del año, y al remplazarse en la ecn. (2) se calcula el desplazamiento del medio día solar en minutos, producido por una rotación terrestre no muy regular.

$B=(n+1) \frac{360}{365}$

Para comprender el comportamiento de la radiación solar extraterrestre $\mathrm{H}_{\mathrm{o}}$ se debe considerar que el Sol es una estrella variable debido a fenómenos como las manchas y fáculas solares, presentes principalmente en las zonas de convección, superficie y atmosfera, provocando que el Sol emita radiación variable a lo largo del año (Peixóto y Oort, 1984). Se conoce que estas variaciones son menores a $\pm 1,5 \%$ sin embargo, la distancia Tierra-Sol varía un 6,7\% a lo largo del año (Gray et al., 2010) y se puede calcular utilizando la ecn. 
(4), que para la mayoría de los propósitos en ingeniería describe la influencia de la distancia Tierra-Sol en la radiación extraterrestre con precisión de un 0,01\% (Duffie y Beckman, 2013). En esta ecuación, Gon es la radiación extraterrestre incidente sobre el plano normal en el enésimo día del año, Gsc corresponde a la constante solar, para este trabajo, con un valor de 1366,8 watts $/ \mathrm{m}^{2}$ y B que incorpora la variación del día del año ecn. (3)

$G_{o n}=G_{s c}(1,000110+0,034221 \cos B+0,001280 \sin B+0,000719 \cos 2 B+0,000077 \sin 2 B)$

El ángulo de la posición del Sol respecto de la normal al plano de incidencia, definido como ángulo cenital $\theta_{z}$, de la siguiente forma $\cos \theta_{z}=\sin \phi \sin \delta+\cos \phi \cos \delta \cos \omega$, donde $\phi$ representa la latitud del lugar de estudio, $\delta$ la declinación solar y $\omega$ la rotación terrestre que define la hora del día. La declinación solar $\delta$ corresponde a la posición angular del Sol al mediodía solar respecto del plano del ecuador, positivo hacia el norte que puede calcularse mediante la ecn. (5), propuesta por Spencer (Duffie y Beckman, 2013; Black et al., 1954) con un error menor de 0,0035 grados, donde B depende del día del año ecn. (3) y $\pi$ corresponde a la constante euclidiana $\mathrm{Pi}$. Obteniéndose la radiación solar extraterrestre incidente sobre la superficie terrestre $\mathrm{H}_{\circ}$, a desde la ecn. (6).

$\delta=(180 / \pi)(0,006918-0,399912 \cos \mathrm{B}+0,070257 \sin \mathrm{B}-0,006758 \cos 2 \mathrm{~B}$

$+0,000907 \sin 2 B-0,002697 \cos 3 B+0,00148 \sin 3 B)$

$H_{\mathrm{O}}=\mathrm{G}_{\mathrm{on}}(\sin \phi \sin \delta+\cos \phi \cos \delta \cos \omega)$

La latitud de la estación meteorológica ESPOCH es $\phi=-1,65^{\circ}$, el tiempo solar se simuló con el ángulo horario $\omega$ que representa el desplazamiento angular del Sol al este o al oeste del meridiano local debido a la rotación de la Tierra, negativo por la mañana y positivo por la tarde, donde el mediodía solar es por definición $\omega=0^{\circ}$.

Los modelos empíricos de transmitancia atmosférica analizados fueron los modelos de Prescott, Hargreaves y García. El primero relaciona la radiación solar con la heliofanía, que fue adaptado del modelo de Ángström, el cual se describe en la ecn. (7) (Baigorria et al., 2004),

$\frac{H}{H_{0}}=a+b \frac{m}{N}$

En dicha ecuación, $\mathrm{H}$ es la medición de radiación total incidente, $\mathrm{H}_{\mathrm{o}}$ es la radiación extraterrestre, m son las horas efectivas medidas con luz solar y $\mathrm{N}$ es el número teórico de horas con luz solar como función de la latitud y la declinación solar, obteniéndose que el día más largo para la localidad es de 12 horas, 5 minutos, 43 segundos, debido a que la latitud es cercana al cero la diferencia entre el día más largo y el más corto es de aproximadamente 11 minutos 27 segundos. Se utilizó $\mathrm{N}$ con un valor de 12 horas para Riobamba a lo largo del año, a y b son coeficientes empíricos donde a corresponde a la radiación difusa en un día nublado, $\mathrm{a}+\mathrm{b}$ a la irradiación global en un cielo despejado (Duran, 2006).

Sin embargo, la confiabilidad de los resultados con la heliofanía se contrapone a la limitada presencia de mediciones de este parámetro en las estaciones meteorológicas en Ecuador. De los modelos basados en parámetros que se obtienen habitualmente en las estaciones meteorológicas convencionales en los países andinos, se considera la propuesta de Bristow y Campbell (Baigorria et al., 2004), que explica la relación entre la diferencia de las temperaturas máximas y mínimas diarias del aire con el calor sensible y calor latente. El calor latente depende de la radiación solar diaria y es el responsable de las temperaturas máximas del aire; además, al anochecer debido a la inercia térmica, el calor sensible gradualmente se pierde en el espacio como radiación de onda larga y otros flujos radiantes, resultando en el decrecimiento de la temperatura hasta alcanzar su valor mínimo justo antes del amanecer. Por esta razón se han propuesto modelos que utilizan las diferencias de temperaturas extremas en el día. Por un lado está el modelo de Hargreaves, que se detalla en la ecn (8), donde se asume una relación dependiente de la raíz cuadrada de la diferencia de temperaturas y por otro lado está el modelo de García, detallado en la ecn (9).

$$
\begin{gathered}
\frac{H}{H_{0}}=a_{h}+b_{h} \Delta T^{0.5} \\
\frac{H}{H_{0}}=a_{g}+b_{g} \frac{\Delta T}{N}
\end{gathered}
$$


Para el cálculo de los coeficientes a y b, se dividieron los datos en dos grupos, las mediciones de temperatura máxima, temperatura mínima y heliofanía. El primer grupo se utilizó para los cálculos de los coeficientes, con información de los años 2009, 2010 y 2011 y el segundo grupo, con información de los años 2007, 2008 y 2012 para la validación de los modelos. Previo al cálculo de los coeficientes, la información de las variables meteorológicas fueron sometidas a análisis de consistencia estadística, identificando errores causados por la transferencia manual de los datos, problemas con los instrumentos, la homogeneidad dimensional y unidades, detección de datos anómalos univariantes, bivariantes y mulivariantes, los datos dudosos fueron analizados individualmente y con herramientas estadísticas se eliminaron datos extremos que no tenían explicación aparente (Peña, 2002), con una confiabilidad superior al 95\%, resultando en una nueva matriz con 1298 datos correspondiendo a días con los parámetros de heliofanía y/o temperaturas máximas y mínimas diarias.

\section{RESULTADOS Y DISCUSIÓN}

Para el cálculo de los coeficientes del modelo de Prescott, de la base de datos disponible, se empleó la información de heliofanía y radiación solar incidente de los años 2009, 2010 y 2011, lo cual corresponde a 1095 registros; sin embargo, por perdidas debido a daños de los equipos, mantenimiento, falla humana, entre otros, aproximadamente el $75 \%$ de los datos fueron útiles. Se verificó que los datos de los años 2009 y los del 2010 cumplen con una distribución normal multivariante y los datos del 2011 cumplen con una distribución univariante, requisito para la detección de datos anómalos. Por otro lado, la validación del modelo fue realizado con la información de los años 2007, 2008 y 2012, que representa aproximadamente el $33 \%$ de la base de datos. Luego del análisis estadístico se obtuvo que más del $94 \%$ de los datos inferidos por el modelo están dentro del rango al 95\% de confiabilidad, con un coeficiente de correlación de 0,81 un error de 0,024 y un coeficiente de determinación de 0,79.

Para el modelo de Prescot ver figura 1, el valor de a fue de 0,18 y el valor de b fue 0,29. Ambas cantidades son menores a los propuestos para toda la región alto andina del Perú, cuyos valores son $a=0,29$ y $b=$ 0,42 según (Baigorria et al., 2004) y a los propuestos por (Black et al., 1954), que son $a=0,23$ y b=0,48. Considerando que a representa la radiación difusa y $a+b$ se equipara a la irradiación global en un cielo despejado, se justifican estos resultados dada la mayor y constante nubosidad en los Andes ecuatorianos respecto al centro y sur de la cordillera andina.

Para el modelo de Hargreaves ver figura 2, luego de la limpieza de datos queda el 67 \% de la información que corresponde a 997 datos, que pertenece a los años 2009, 2010 y 2011 . Al realizar la regresión con este modelo se obtuvieron valores de $a_{h}=-0,11$ y $b_{h}=0,12$, con un coeficiente de determinación de 0,48 y un error de 0,118 . En la validación de los coeficientes se emplearon los 502 datos restantes de los años 2007,2008 y 2012 , obteniéndose que más del $94 \%$ de los datos estén dentro del rango a un $95 \%$ de confiabilidad y un coeficiente de correlación de 0,67.

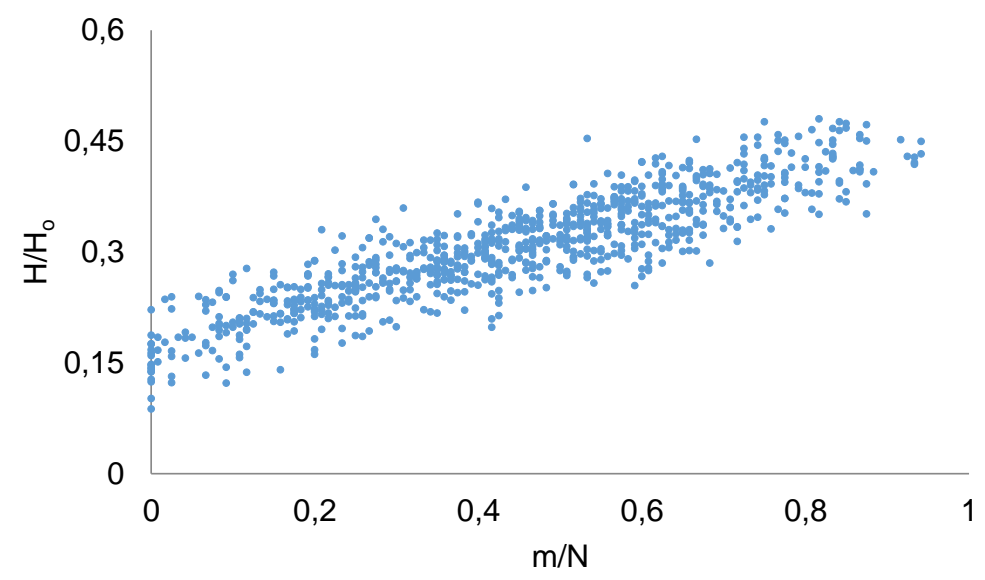

Fig. 1: Transmitancia atmosférica en función de la heliofanía- Modelo de Prescot 


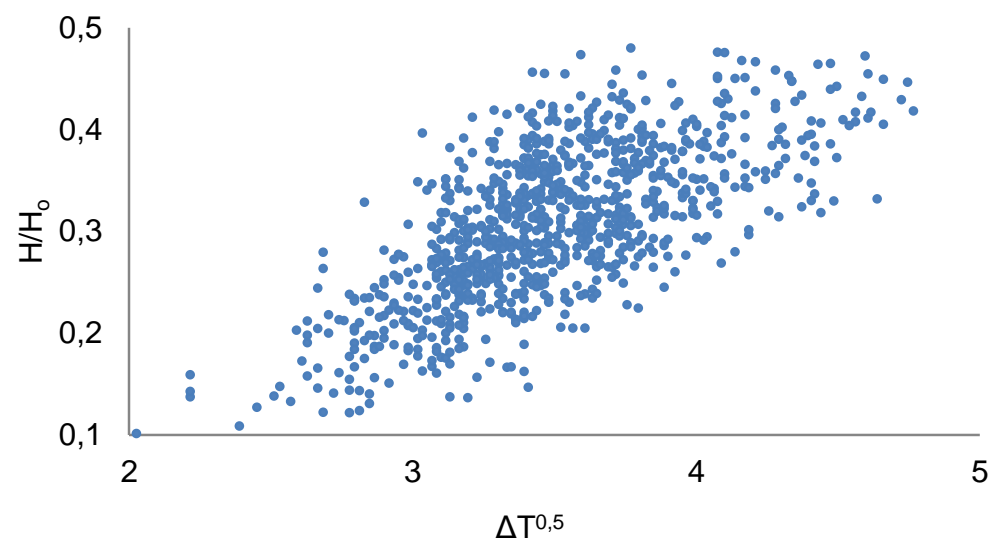

Fig. 2: Transmitancia atmosférica en función de la diferencia de temperaturas- Modelo de Hargreaves

En tercer lugar, con el modelo de García ver figura 4, se obtuvieron los siguientes coeficientes: $a_{g}=0,10$ y $b_{g}=0,20$, con un coeficiente de determinación de 0,46 y un error de 0,01 mostrando un comportamiento similar con el propuesto para los andes peruanos $a_{g}=0,457$ y $b_{g}=0,207$ dado que los coeficientes $b_{g}$ tienen valores muy cercanos; sin embargo, ag presenta una importante diferencia.

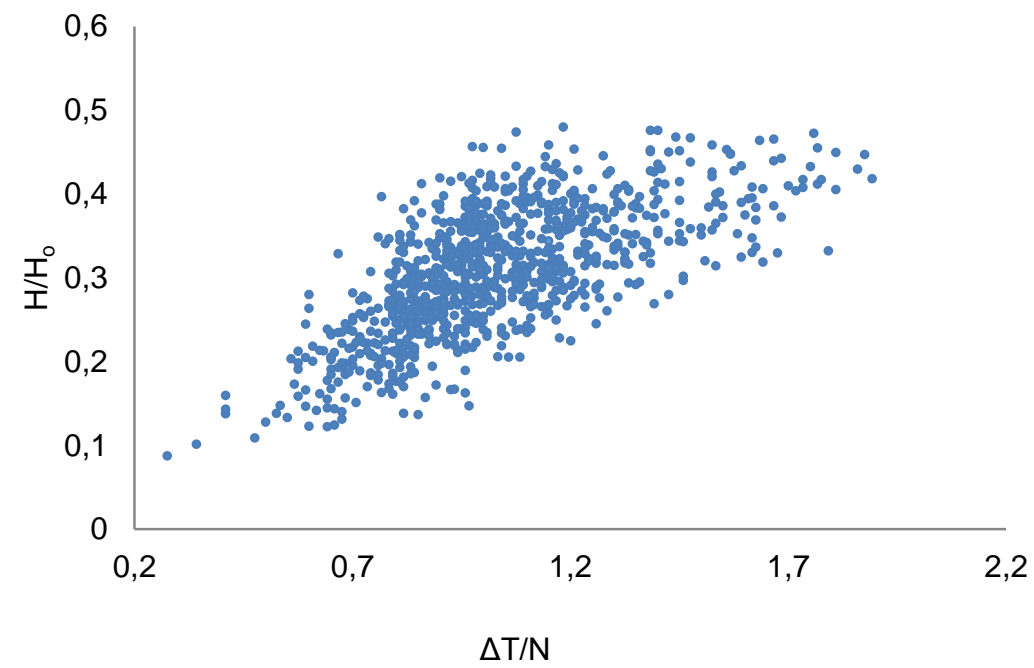

Fig. 3: Transmitancia atmosférica en función de la diferencia de temperaturas- Modelo de García.

Finalmente, para la validación de los coeficientes de todos los modelos, se emplearon un $33 \%$ de los datos que fueron tomados entre los años 2007, 2008 y 2012. Luego del análisis estadístico, se estableció que algo más del $94 \%$ de los datos estuvieron dentro del rango a un $95 \%$ de confiabilidad y un coeficiente de correlación de 0,66. Un resumen de los resultados se muestra en la tabla 1.

Tabla. 1: Comparación de resultados

\begin{tabular}{|c|c|c|c|c|c|c|}
\hline Modelo & a & B & $\begin{array}{c}\text { Coeficiente de } \\
\text { Determinación }\end{array}$ & $\begin{array}{c}\text { Error } \\
\text { (modelo) }\end{array}$ & $\begin{array}{c}\text { Confiabilidad } \\
\text { (validación) }\end{array}$ & $\begin{array}{c}\text { Coeficiente } \\
\text { Correlación }\end{array}$ \\
\hline Prescott & 0,18 & 0,29 & 0,79 & 0,024 & $94 \%-95 \%$ & 0,81 \\
\hline Hargreaves & $-0,11$ & 0,12 & 0,48 & 0,118 & $94 \%-95 \%$ & 0,67 \\
\hline García & 0,10 & 0,20 & 0,46 & 0,01 & $94 \%-95 \%$ & 0,66 \\
\hline
\end{tabular}

\section{CONCLUSIONES}

El análisis inferencial multivariante permitió separar los datos atípicos inmersos en la muestra, obteniendo relaciones aproximadamente lineales entre las variables meteorológicas medidas y calculadas en el estudio. El coeficiente de correlación del modelo de Prescott es el más alto de todos, con un valor de 0,81 y un error de 0,024 para la relación entre la radiación solar incidente y la heliofanía. Por este motivo, los coeficientes de este modelo son recomendados para la ciudad de Riobamba y es una clara muestra que las relaciones 
con heliofanía estiman mejor la radiación solar incidente que las relaciones con diferencias de temperatura extremas.

Debido a que el de modelo de García presentó un menor error y un coeficiente de correlación ligeramente menor que el modelo de Hargreaves, por lo que, de no contarse con la información de heliofanía, el modelo de García es el adecuado para la ciudad de Riobamba.

Finalmente, al hacer una comparación de los coeficientes con otros autores, a pesar de ciertas similitudes tales como estar en la zona andina o a una altura similar, no es posible generalizar los valores de los coeficientes, puesto que las condiciones de cada región hacen que los valores de transmisividad de la atmósfera varíen sustancialmente.

\section{AGRADECIMIENTOS}

Los autores agradecen a la SENESCYT por crear las condiciones para desarrollar ciencia en el Ecuador, al INER por la confianza en el Grupo Energías Alternativas y Ambiente de la ESPOCH y a la fundación italiana CDF por su tradicional apoyo.

\section{REFERENCIAS}

Baigorria, G. y otros tres autores. Atmospheric transmissivity: distribution and empirical estimation around the central Andes. International Journal of Climatology, DOI: 10.1002/joc.1060 (en línea). 24, 1121-1136 (2004). http://onlinelibrary.wiley.com/doi/10.1002/joc.1060/pdf. Acceso 23 de enero 2014.

Black, J., Bonython, C., Prescott, J. Solar radiation and the duration of sunshine. Q.J.R. Meteorol. Soc. doi: 10.1002/qj.49708034411 (en línea). 80, 231-235 (1954).

http://onlinelibrary.wiley.com/doi/10.1002/qj.49708034411. Acceso 23 de enero 2014.

Duffie, J., Beckman, W. Solar Engineering of Thermal Processes, Fourth Edition, 2-133, John Wiley \& Sons, Inc. New Jersey, USA, 2013.

Duran, A. A new formulation for solar irradiation and sunshine duration estimation. International journal of energy research. DOI: 10.1002/er.1229 (en línea). 31, 109-118 (2006).

http://onlinelibrary.wiley.com/doi/10.1002/er.1229. Acceso 23 de enero 2014.

Gray, L. y otros doce autores, Solar influences on climate, Rev. Geophys., doi:10.1029/2009RG000282 (en línea). 48, $1-53$ (2010). http://onlinelibrary.wiley.com/doi/10.1029/2009RG000282/abstract. Acceso 20 agosto 2013.

Igbal, M. An Introduction to Solar Radiation. 1st Edition, vol 1, 1-34, Academic Press Inc., New York, USA, (1983).

Machado, A. y otros tres autores. Estudio del Secado de Anacardo (Anacardium occidentale L.) mediante Secador Solar de Radiación Directa. Información Tecnológica, ISSN 0718-0764 (en línea), 21(1), 31-37 (2010). http://www.scielo.cl/scielo.php?script=sci_arttext\&pid=S0718-07642010000100006\&lng=es\&nrm=iso. Acceso 1 mayo 2014.

Magnani, F. y otros tres autores. Growth patterns of Pinus sylvestris across Europe: a functional analysis using the HYDRALL model. iForest - Biogeosciences and Forestry, ISSN: 1971-7458 (en línea), 2(5), 162171 (2009). http://www.sisef.it/iforest/pdf/?id=ifor0516-002. Acceso 10 agosto 2014.

Monne, C., Alonso, S., Palacin, F. Evaluación de una Instalación de Refrigeración por Absorción con Energía Solar. Información Tecnológica, ISSN 0718-0764 (en línea), 22(3), 39-44 (2011). http://www.scielo.cl/scielo.php?script=sci_arttext\&pid=S0718-07642011000300006\&lng=es\&nrm=iso.

Acceso 20 agosto 2014.

Lara, F. y otros tres autores. Metodología para el Dimensionamiento y Optimización de un Concentrador Lineal Fresnel. Información Tecnológica, ISSN 0718-0764 (en línea), 24(1), 115-128 (2013). http://www.scielo.cl/scielo.php?script=sci_arttext\&pid=S0718-07642013000100013\&lng=es\&nrm=iso.

Acceso 1 de mayo 2014.

Lema, A. y otros tres autores. Análisis del Efecto de Variables de Diseño en el Comportamiento de una Secadora de Granos de Amaranto. Información Tecnológica, ISSN 0718-0764 (en línea), 16(5), 43-48 
(2005). http://www.scielo.cl/scielo.php?script=sci_arttext\&pid=S0718-07642005000500008\&lng=es\&tlng=es. 10.4067/S0718-07642005000500008. Acceso 20 agosto 2014.

Peixóto, J., Oort., A. Physics of climate. Rev. Mod. Phys. DOI 10.1103/RevModPhys (en línea), 56, 365-430 (1984). http://link.aps.org/doi/10.1103/RevModPhys.56.365. Acceso 22 mayo 2013.

Peña, D. Análisis de datos multivariantes. Primera edición, vol 24, Editorial McGraw-Hill. Madrid, España, (2002).

Raichijk, C., Gallegos G., Righini R. Evaluación de un método alternativo para la estimación de valores medios mensuales de irradiación global en argentina. Avances en Energías Renovables y Medio Ambiente, ISSN 0329-5184 (en línea), 9, 5-8 (2005). http://www.asades.org.ar/modulos/averma/trabajos/2005/2005t011-a002.pdf. Acceso 20 abril 2013.

Recalde, C., Echeverría, M., Castro, R. Descomposición de Materia Orgánica con Microorganismos Benéficos Magnetizados. Información Tecnológica, ISSN 0718-0764 (en línea), 24(6), 9-16 (2013). http://www.scielo.cl/scielo.php?script=sci_arttext\&pid=S0718-07642013000600003\&lng=es\&nrm=iso.Acceso Acceso 5 enero 2014.

Recalde, C. y otros cinco autores. Los Tesoros del Qhapaq Ñan. Primera edición, vol 1, 85-112, Editorial el Conejo. Riobamba, Ecuador, (2010).

Rodríguez, C., Sarmiento, A. Dimensionado mediante simulación de sistemas de energía solar fotovoltaica aplicados a la electrificación rural, Ingeniería Mecánica, ISSN 1815-5944 (en línea). 14, 13-21 (2010). Disponible en http://www.ingenieriamecanica.cujae.edu.cu/index.php/revistaim/article/view/. Acceso 21 mayo 2013.

Salazar, G., Saravia, L., Romero, G. Comparación de datos esporádicos de irradiación medidos simultáneamente en dos localidades de la provincia de Salta. Avances en Energías Renovables y Medio Ambiente. ISSN 0329-5184 (en línea), 11, 73-79 (2007).

http://www.asades.org.ar/modulos/averma/trabajos/2007/2007-t011-a011.pdf. Acceso 24 mayo 2013.

Sayago, S. y otros tres autores. Radiación solar horaria: Modelos de estimación a partir de variables meteorológicas básicas. Avances en Energías Renovables y Medio Ambiente, ISSN 0329_5184 (en línea), 15, 51-57 (2011). http://www.asades.org.ar/modulos/averma/trabajos/2011/2011-t011-a008.pdf. Acceso 10 febrero 2013.

Vernich, L. y Zuanni, F. About the minimum number of years required to stabilize the solar irradiation statistical estimates. Solar Energy. ISSN 0038-092X (en línea), 57(6), 445.447 (1996). http://dx.doi.org/10.1016/S0038-092X(96)00121-1. Acceso 1 de mayo 2014.

Villeda, G. y otros tres autores. Seguidor Solar de Dos Ejes para un Horno Solar. Información Tecnológica, ISSN 0718-0764 (en línea), 22(2), 95-106 (2011).

http://www.scielo.cl/scielo.php?script=sci_arttext\&pid=S0718-07642011000200011\&lng=es\&nrm=iso.

Acceso 1 de mayo 2014. 